\title{
Diversity of Microbial Signatures in Asthmatic Airways
}

This article was published in the following Dove Press journal:

International Journal of General Medicine

\author{
Aisha Alamri (D) \\ Department of Clinical Laboratory \\ Sciences, College of Applied Medical \\ Sciences Imam Abdulrahman Bin Faisal \\ University, Dammam, Kingdom of Saudi \\ Arabia
}

\begin{abstract}
Asthma is a chronic inflammatory disease affecting the respiratory system. The global incidence of asthma is rising. Clinical and experimental models of asthma clearly indicate that the disease is multifactorial in nature with a wide array of factors contributing to progression and exacerbation, including interactions between immunological markers and the microbial community populating the respiratory tract. In particular, strict hygiene compliance during the early years of life and early exposure to antibiotics are linked to alterations in the biological environment within the airways and to changes in immunological markers, leading to allergies, such as asthma. With the gap in current research knowledge on the various non-bacterial microbial communities in the asthmatic airways, this review summarizes current methods used to assess microbial diversity as well as evidence for the link between microbial alterations and asthma, including changes in the bacterial microbiome, often characterized by the outgrowth of certain bacterial phyla such as proteobacteria and Firmicutes, in addition to disrupted mycobiome, virome, and parasitome. The current review emphasizes the dynamic, context-dependent changes in the microbiome in asthma and the importance of broad-scope analyses, covering a wide range of taxa. In conclusion, the interaction between the resident microbiota and the immune system is essential and significant in modulating the inflammatory responses; however, further investigations are needed to improve our understanding of the risk factors that disrupt the diversity of the microbiome in the different body systems.
\end{abstract}

Keywords: microbiome, mycobiome, virome, parasitome, dysbiosis, microbiota

\section{Introduction}

Globally, it is estimated that more than 300 million people are affected by the different phenotypes of asthma. ${ }^{1}$ It is essential to investigate the factors that may lead to asthma exacerbation, particularly among high-risk individuals. ${ }^{2}$ Risk factors contributing to the occurrence and progression of asthma include environmental and genetic factors that contribute to alterations in the respiratory ecosystem. $^{2}$

There is growing evidence that microbial communities populating the airways during the early years of life are vital for immune system development and for the regulation of inflammatory responses. ${ }^{3}$ The human microbiome refers to the overall microbial genomes in the human body. Microbiota, a term often used interchangeably with microbiome, is frequently used to describe the microorganisms colonizing the inner and outer body surfaces. ${ }^{4}$ Dysbiosis, or an imbalance in the distribution of resident microbial taxa, has been linked to the development of various allergic and inflammatory conditions, including asthma. ${ }^{3}$ The term dysbiosis has been used increasingly in the last decade and
Correspondence: Aisha Alamri

Email aiamri@iau.edu.sa
International Journal of General Medicine 2021:14 1367-1378

(c) 2021 Alamri. This work is published and licensed by Dove Medical Press Limited. The full terms of this license are available at https://www.dovepress.com/terms.php C. you hereby accept the Terms. Non-commercial uses of the work are permitted without any further permission from Dove Medical Press Limited, provided the work is properly attributed. For permission for commercial use of this work, please see paragraphs 4.2 and 5 of our Terms (https://www.dovepress.com/terms.php). 
is the basis for many studies of the interactions between the human microbiome and various inflammatory conditions, with more than 2370 published articles in 2020 alone (265, Asthma related), compared to 34 published work in 2010. (NCBI.com, last accessed January 27, 2021).

Advances in molecular biology technology have led to a wide array of studies of allergic conditions using metagenomics, transcriptomics, proteomics, and, more recently, metabolomics approaches. In particular, metabolomics target microbial metabolites generated at the site of the inflammatory reaction, thus providing a relatively "'functional" explanation of the disease condition $^{5}$ (Figure 1).

In this review, evidence for the interaction between asthma progression and the resident microbiota of the airway is summarized. In particular, the current review provides a brief description of sampling and analysis types, followed by a comprehensive overview of key findings. Since the microbiome represents a consortium of diverse microbial taxa and is not restricted to bacteria, this review describes studies of the bacterial, fungal, and viral components of the microbiome in relation to asthma. Finally, challenges and key areas for future research are highlighted.

\section{Sampling Strategies for Asthmatic Microbiome Analyses}

Culture-based approaches to assess the microbial burden in various body sites are limited by the difficult recovery of atypical bacteria and fastidious organisms with complex nutritional requirements. ${ }^{6}$ Molecular techniques provide a reliable alternative to culture-based approaches. Rapid technological advances have allowed for the generation of more meaningful and impactful data related to the diversity of the microbiome at the DNA or RNA level. ${ }^{7}$

The vast majority of studies of bacterial diversity in different body compartments have used 16S rRNA pyrosequencing techniques. The $16 \mathrm{~S}$ ribosomal RNA gene is a 1500-bp housekeeping gene that has historically been used for studies of bacterial phylogeny and taxonomy. This gene contains nine hypervariable regions (V1-V9) with high levels of sequence diversity among bacterial taxa, thus allowing species identification and differentiation. ${ }^{8}$ However, this approach is limited by the high similarity of $16 \mathrm{~S}$ rRNA gene within phyla or genera and low precision; additionally, searches are restricted to the level of identification and therefore additional information about the presence of certain virulence or antibiotic

\begin{tabular}{|c|c|c|c|}
\hline Approach & Description & Target & Platform \\
\hline Amplicon Sequencing & $\begin{array}{l}\text { Sequencing of the highly conserved genes that serve as a marker for } \\
\text { the microbial genus and species. The technique is reliable, has low- } \\
\text { cost and widely used in microbiome studies to assess the bacterial } \\
\text { and fungal diversity in clinical and environmental samples. This } \\
\text { approach is limited to taxonomy profiling only and results generated } \\
\text { are relative and not absolute quantities. }\end{array}$ & $\begin{array}{l}\text { 16S rRNA bacteria } \\
18 S \text { rRNA, ITS Fungal species } \\
\text { 18S rRNA parasitic species }\end{array}$ & $\begin{array}{l}\text { Sanger-sequencing, } \\
\text { Pyrosequencing, } \\
\text { Next generation sequencing }\end{array}$ \\
\hline Shot-gun metagenomics & $\begin{array}{l}\text { Examining the collective microbial genomes in a sample by trimming } \\
\text { DNA extracted from the sample and sequencing the small } \\
\text { fragments. It requires a reference genome and a high throughput } \\
\text { computer programs to assemble the data. } \\
\text { Risk of generating false positive data is high due to errors during } \\
\text { sequence assembly }\end{array}$ & $\begin{array}{l}\text { All genomic content of the } \\
\text { microorganisms in a sample. }\end{array}$ & Next-generation sequencing \\
\hline Transcriptomics & $\begin{array}{l}\text { It allows for identification of differential gene expression in the cells; } \\
\text { naturally or in response to certain health conditions/environmental } \\
\text { factors. However, no further information on the protein product can } \\
\text { be obtained }\end{array}$ & All/targeted mRNA transcripts & $\begin{array}{l}\text { Next-generation sequencing } \\
\text { (RNA-Seq), Microarray, } \\
\text { NanoString Gene expression } \\
\text { technology }\end{array}$ \\
\hline Proteomics & $\begin{array}{l}\text { Gene activity can be evaluated and significant biological information } \\
\text { for many physiological and health problems can be assessed. }\end{array}$ & $\begin{array}{l}\text { All/targeted Protein products of } \\
\text { the microbial genes }\end{array}$ & Mass spectrometry \\
\hline Metabolomics & $\begin{array}{l}\text { High throughput analysis of metabolites (bi-products) released by } \\
\text { microorganisms in biological systems can be studied. }\end{array}$ & $\begin{array}{l}\text { Useful to compare metabolites } \\
\text { and disease biomarkers in } \\
\text { different health conditions such } \\
\text { as allergies }\end{array}$ & $\begin{array}{l}\text { Mass spectrometry, } \\
\text { Nuclear Magnetic Resonance } \\
\text { (NMR) spectroscopy }\end{array}$ \\
\hline
\end{tabular}

Figure I Description of multi-omics technology used to analyze microbial communities residing in the different biological samples. 
resistance genes is not readily obtained. ${ }^{9}$ Although wholegenome shotgun sequencing is challenging and requires complex analysis software, it is a precise alternative for species identification and other genomic analyses. ${ }^{9}$

Although asthma is localized in the respiratory tract, extrapulmonary samples have been used to assess microbial diversity in patients, including stool samples, rectal swabs, gastric secretions samples, and, more recently, blood samples. ${ }^{10-12}$ Oral and upper respiratory tract samples, such as saliva, throat, nose, and nasopharyngeal swabs, are widely used. Lower respiratory tract samples, such as bronchoalveolar lavage (BAL), bronchial aspirates, brushings, biopsies, and sputum (induced or spontaneous), are more representative of the respiratory microbiome, ${ }^{13}$ however, the invasive nature of the procedures makes sample collection from children impractical. ${ }^{14}$ Moreover, microbial diversity varies considerably among different respiratory compartments in the same individual. $^{3}$

\section{Development of the Respiratory Microbiome During Childhood}

The in utero environment has long been considered a sterile environment. However, there is evidence for early bacterial colonization in the placenta, fetal membranes, amniotic fluid, cord blood samples, and meconium of full-term healthy infants. ${ }^{15,16}$ Although there are reports of pathogenic alterations in the airway microbiota in lung diseases, such as chronic obstructive pulmonary disease (COPD) and cystic fibrosis, it is not clear how the airway microbiome develops in newborns. ${ }^{17}$

Developmentally immature lungs make premature infants susceptible to injuries that could lead to pulmonary alveolar and vascular dysfunction or even bronchopulmonary dysplasia (BPD). The progression of BPD is largely associated with inflammatory reactions in the airways, as evidenced by elevated levels of inflammatory markers. Therefore, it is generally thought that early interactions involving the airway microbiome may prime the development of the pulmonary immune system and that dysbiosis during development may set the stage for subsequent lung disease. ${ }^{18}$

Studies of the airway microbiota in infants have concluded that there is a distinct oral microbiome signature at birth in premature and full-term infants, and the microbial composition of the airway is different and less diverse in infants with BPD than in full-term and extremely low-level birth-weight infants. ${ }^{18}$ Interestingly, temporal changes in the airway microbiome have been detected in extremely low-level birth-weight infants, characterized by a significant increase in Proteobacteria and decreases in Firmicutes, Actinobacteria, Bacteroides, and Fusobacteria over time. ${ }^{18}$ Although it is generally believed that airway colonization in infants originates during delivery in the birth canal, some studies have found similar microbiome patterns among neonates born via the vagina and caesarean section, suggesting a transplacental route of colonization. ${ }^{19}$

Similarly, Mourani et al analyzed serially collected tracheal aspirates over a 21-day period from 10 preterm infants with different degrees of BPD who were under mechanical ventilation. ${ }^{20}$ Evidence for airway microbial colonization was more prominent after 72 hours and Staphylococcus, Ureaplasma parvum, and Ureaplasma urealyticum were dominant, with low abundances of Pseudomonas, Enterococcus, and Escherichia. ${ }^{20}$

It should be noted that the microbiome of the lower airway, though difficult to access, is more representative of lung pathologic conditions. Therefore, further analyses of the lower airway microbiome are needed to improve our understanding of the factors that lead to the progression of BPD. ${ }^{3}$

In a study of 25 intubated preterm infants, the subset of infants who developed BPD exhibited reduced diversity of the airway microbiome at birth, suggesting a potential link between microbial diversity and quantity and the progression of BPD; Acinetobacter was the dominant genus observed in preterm infants. ${ }^{21}$

Studies have also suggested that respiratory microbiome does not stabilize during infancy but over a longer time period. $^{22,23}$ In one study, 827 nasal swabs from 47 infants collected every 2 weeks revealed an increase in the microbial density mirrored by a reduced diversity within the first year of life; the observed differences among individuals in the microbiome suggest that there is a ""personalized" respiratory microbiota. ${ }^{22}$ This study also concluded that age and seasonality shape the individual microbiome.

Nasopharyngeal microbiota profiles for 60 healthy children at six weeks, six, 12, and 24 months of age analyzed by $16 \mathrm{~S}$ rRNA pyrosequencing revealed eight distinct respiratory microbial profiles by the age of 6 weeks, with the early presence of certain genera, such as Moraxella and Corynebacterium/Dolosigranulum. Furthermore, less stable profiles were marked by the high abundance of Haemophilus or Streptococcus. ${ }^{23}$ 
Studies of the development of fungal communities in early infancy and childhood are scarce, with few analyses of the intestinal mycobiome. ${ }^{24}$ Infant gastrointestinal tracts are mainly colonized by Saccharomyces and Malassezia. ${ }^{24}$ However, the lack of longitudinal studies of changes in fungal communities in infants makes it difficult to identify certain genera as predictors of allergic conditions later in life.

\section{Bacterial Microbiome}

\section{Bacterial Microbiome in the Airways}

The relationship between alterations in the airway microbiome and respiratory allergic conditions has been analyzed using many sample types and sites within the airways, including upper respiratory tract samples (nose, nasopharynx, oropharynx throat, and saliva) and lower respiratory tract samples (sputum (expectorant or induced), bronchial aspirates, and bronchoalveolar lavage). ${ }^{25,26}$

The microbiome in the saliva is shed from various niches in the oral cavity and appears to be representative of the overall oral microbiome. Although the majority of studies targeting the salivary microbiome have focused on its relationship with dental diseases, a subset of studies have assessed the relationship between the salivary microbiome and allergic disorders, such as asthma. ${ }^{25,27}$ In one study, saliva was collected longitudinally at three, six, 12, and 24 months and seven years from children with allergies and healthy counterparts. Allergies, particularly asthma, resulted in alterations in the microbial composition with reduced diversity at the age of seven years, suggesting the presence of an impaired immune response during infancy. ${ }^{25}$ Of note, increases in the relative abundance of certain species, such as Gemella haemolysans, in the saliva of children with allergies were mirrored by an increased abundance of Lactobacillus species in healthy individuals, suggesting that lactic acid bacteria contribute to the development of an equilibrium state in the oral mucosa and thus to a reduction in allergic attacks. $^{25}$

Espuela-Ortiz et al examined the salivary microbial composition in 57 African-American children with and without asthma based on 16S RNA gene profiles and detected differences between cases and controls with respect to diversity and relative abundance, with significantly higher frequencies of Streptococcus and Veillonella in the disease group than in the control group. ${ }^{27}$
Other studies have examined the microbial community in the throat of patients with asthma. Based on 327 throat swabs evaluated by pyrosequencing of the 16S rRNA gene, there was no difference in the oral microbiome between cases and controls. ${ }^{26}$ However, in an analysis of 68 nasal swabs from the two groups, a significant difference in the microbial community was found between children with asthma and healthy controls, with a lower diversity and a predominance of Moraxella. ${ }^{26}$ The throat microbiota has also been examined in a subset of children with cystic fibrosis and asthma in addition to healthy children aged six to 12 years. Despite the presence of a similar core microbiome in both groups, including Prevotella, Streptococcus, Neisseria, Veillonella, and Haemophilus, a higher abundance of certain opportunistic pathogens, particularly Pseudomonas, Staphylococcus, and Streptococcus, was noted in the asthmatic group, suggesting that dysbiosis plays a major role in the progression of asthma in patients with cystic fibrosis. ${ }^{28}$ The defective mucociliary clearance mechanism enhances the overgrowth of certain microbial taxa and with the accumulation of toxins and metabolites, an inflammatory reaction is triggered leading to signs and symptoms of asthma.

The nose is colonized by potentially pathogenic, opportunistic organisms that can spread to various locations in the respiratory tract and may be involved in the development of allergic conditions, such as allergic rhinitis, chronic rhinosinusitis (CRS), acute respiratory tract infections, otitis media, and asthma. ${ }^{29,30}$ Previous studies of the nasal microbiota of patients with chronic rhinitis have reported significant reductions in Corynebacterium and Peptoniphilus. ${ }^{31,32}$ Furthermore, the diminished colonization of certain clostridia may promote $\mathrm{T}$ helper type 2 (TH2) inflammation and consequently upper respiratory tract inflammation. ${ }^{32}$

Interestingly, the nasal microbiota exhibits different alterations among the different phenotypes of asthma. When the nasal microbiota of 163 children with different asthma phenotypes were assessed, a common core genera was present in more than $90 \%$ of the tested children, including Moraxella, Haemophilus, Staphylococci, and Streptococcus; however, Proteobacteria, Actinobacteria, Bacteroides, Corynebacterium, Dolosigranulum, and Prevotella varied considerably across asthma phenotypic clusters, suggesting that the respiratory microbiota is an effective biomarker that may aid in clinical classification. ${ }^{33}$ Based on a metagenomics analysis of patients with asthma and healthy controls, the healthy group within the elderly 
category ( $>65$ years of age) showed a significant increase in Proteobacteria, whereas young, healthy individuals showed elevations in Propionibacterium and Corynebacteriales. Among the asthmatic groups, Staphylococcus, Propionibacterium, and Moraxella were more frequent in the young patients than in elderly patients, suggesting that age contributes to the relative abundances of resident taxa, even when the diagnosis of asthma is established. ${ }^{34}$

Based on 16S rRNA pyrosequencing, the relative abundances of Streptococci and Burkholderia are higher in patients with asthma who have comorbid CRS than in those without CRS. ${ }^{35}$ Interestingly, patients with frequent emergency room visits due to asthma exacerbation tended to have a higher relative abundance of Proteobacteria than that of patients with asthma who visited the emergency room less frequently ${ }^{35}$ (Figure 2).

The severity of asthma seems to influence the microbial community composition in the airway. When asthmatic adults with asthma exacerbation $(\mathrm{n}=20)$ were compared with 31 subjects with asthma with no exacerbation, certain taxa were common to both groups (particularly Bacteroides and Proteobacteria); however, for some taxa, including Prevotella buccalis, Dialister invisus, Gardnerella vaginalis, and Alkanindiges hongkongensis, abundance was correlated with the severity of asthma. ${ }^{36}$

The lung is constantly exposed to the microbiota either by inhalation or subclinical micro-aspiration from birth. Historically, lungs have been believed to be sterile environment, and this dogma has persisted in contemporary medicine for many years. In the last decade, our understanding of interactions between the lung and microbiota has progressed substantially. This shift in perspective stems from new knowledge that the moist and warm lung environment is constantly exposed to microbes by inhalation. In addition, the resident microbiota in the oropharynx is frequently exposed to the lung environment and subclinical aspiration is a common phenomenon; due to the outstanding ability of microbial species to adapt to new environments, a wide range of bacteria might colonize the lung tissues. $^{37}$ This notion is supported by recent studies that have identified "constantly present" microbial species using special collection techniques, such as bronchoscopy, to collect BAL. ${ }^{38}$ The microbiota of the right upper lobe more closely resembled that of the upper respiratory tract than did the microbiota from more distal sites, supporting the notion that microbial species frequent migrate to new, harsher, yet lesspopulated locations. ${ }^{39}$

Moreover, studies of children with asthma have revealed that the bronchial tree contains over 2000 bacterial genomes per $\mathrm{cm}^{2}$ of surface. Pathogenic proteobacteria (Haemophilus species, in particular) are more frequent among adults and children with asthma and Bacteroidetes, (Prevotella species) are more frequent in healthy, non-asthmatic subjects, suggesting that the microbiota is significantly altered in disease conditions. ${ }^{40}$ A positive correlation between the severity of asthma and the dominance of Proteobacteria has also been identified, with a more diverse microbial environment in asthmatic airways. ${ }^{40,41}$ However, there are limited data regarding the role of the lung microbiota in the regulation of immunity and homeostasis. ${ }^{38}$

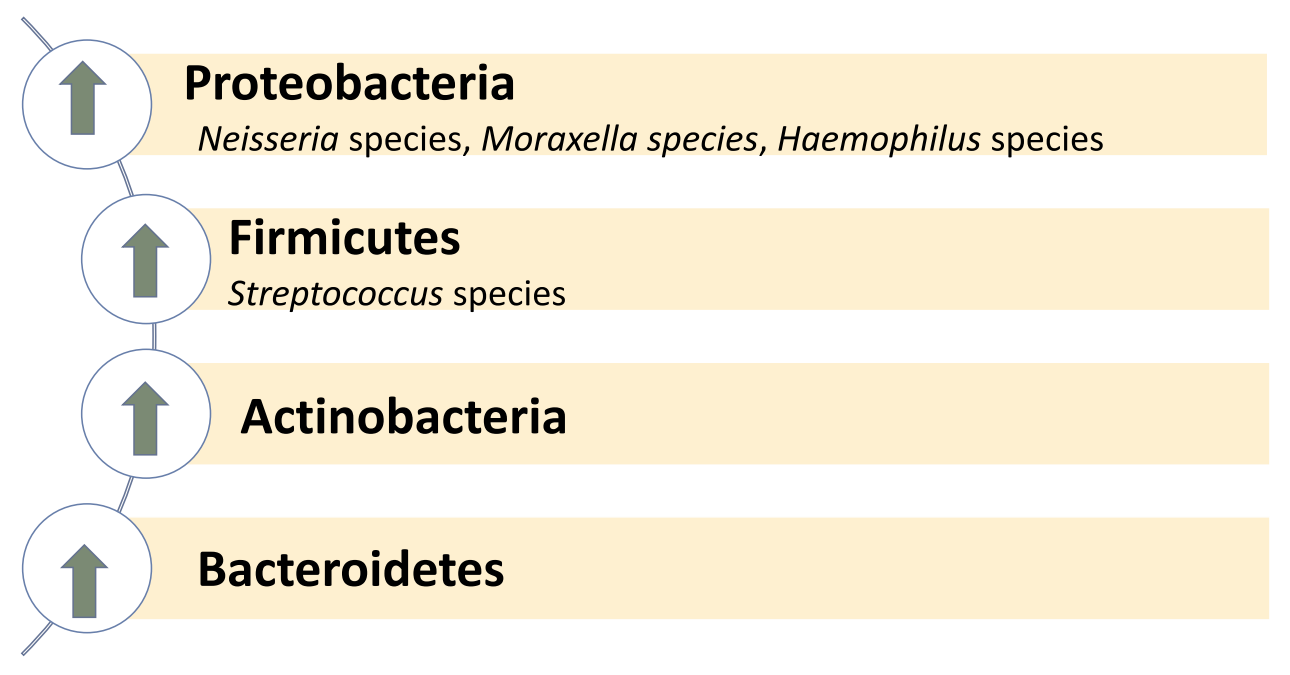

Figure 2 Bacterial phyla and genera that appear to outgrow in asthmatic airways. 


\section{Intestinal (Gut) Microbiome}

Among microbiome studies, the vast majority are focused on the gut microbiome, with trillions of microbial genomes, compared with relatively few studies of the airway's microbiome. More recently, the gut-lung axis has been used for broader analyses of allergic respiratory conditions. ${ }^{42}$

A growing body of evidence suggests that the development of the gut microbiome during infancy is a "critical window" in which disturbances at the level of microbial abundance and diversity are a major predictor of asthma. ${ }^{17}$ In 319 subjects enrolled in a longitudinal, multicenter study, the risk of asthma was linked to transient gut microbial dysbiosis during the first 100 days of life. Notably, bacterial genera, such as Lachnospira, Veillonella, Faecalibacterium, and Rothia, were significantly decreased in children at risk of asthma. ${ }^{17}$ When germfree mice were treated with strains of these genera to restore the microbial balance, airway-induced inflammation declined dramatically. ${ }^{17}$

\section{Mycobiome}

Although the majority of studies of microbial diversity in the airways have addressed only the bacterial composition, the fungal microbiome (mycobiome) component represents an essential element of the total microbial communities in the human body. To study the diversity of fungal communities, the internal transcribed spacer (ITS1, 2) regions is generally used in addition to $18 \mathrm{~S}$ rRNA. ${ }^{25}$

\section{Airway Mycobiome}

Various fungi have been shown to exacerbate asthma by colonizing the airways for long periods and by producing toxins, enzymes, and a wide range of allergenic proteins. ${ }^{43}$ Additionally, colonization by fungal species does not always reflect the presence of pathogenic conditions, as many fungal species coexist with bacterial taxa naturally in the oral cavity. ${ }^{44}$ Analyses of the salivary mycobiome in healthy individuals have demonstrated the presence of a "'basal mycobiome" in the oral cavity, including a mixture of fungal species predominated by Candida species, followed by Cladosporium, Aureobasidium, Saccharomycetales, Aspergillus, and Cryptococcus. ${ }^{44}$

In the nasal mycobiome of patients with allergic rhinitis, over 69 common genera have been detected; Aspergillus, Cryptococcus, and Alternaria are closely linked to respiratory symptoms. Additionally, the genus
Malassezia is prominent in both case and control cohorts. ${ }^{45}$ Additionally, analyses of the nasal mucosa have revealed a cluster of three genera (Cladosporium, Penicillium, and Aspergillus) that are dominant in individuals with respiratory allergies, whereas the frequency of Alternaria alternata is low in patients with allergies. ${ }^{46}$

The inhalation of fungal spores can result in fungal deposition in the airways. In healthy lung tissues, these potentially allergenic spores are cleared as part of the natural lung defense mechanism. ${ }^{47}$ However, in a subset of patients with chronic respiratory dysfunction, such as patients with COPD or asthma, spores can germinate and persist for long periods, thereby increasing the fungal burden and contributing to chronic fungal infections, such as chronic pulmonary aspergillosis. ${ }^{47}$

Using induced sputum extracted from patients with asthma $(n=30)$ and healthy controls $(n=13)^{48}, 136$ fungal species were identified, 90 of which were more frequent in patients with asthma than in controls, including Psathyrella candolleana, Termitomyces clypeatus, Grifola sordulenta, and Malassezia pachydermatis, and 46 of which were predominant in healthy controls, including Eremothecium sinecaudum, Systenostrema alba, Cladosporium cladosporioides, and Vanderwaltozyma polyspora. ${ }^{48}$ Interestingly, Malassezia pachydermatis, a frequent cause of atopic dermatitis, was also common in the asthmatic fungal collection. ${ }^{48}$

In another study, ITS region 1 was examined in bronchoalveolar lavage extracted from a subset of patients with different respiratory manifestations, including severe and mild asthma, and from control healthy subjects. A mycobiome analysis revealed a higher fungal load among patients with asthma than in controls, with a high abundance of the Aspergillus fumigatus complex. Interestingly, corticosteroid therapy was identified as a major risk factor for an increased fungal burden in asthmatic airways. ${ }^{49}$

In the lower airway mycobiome based on sputum samples, untreated patients with asthma showed increases in Wallemia, Mortierella, and Fusarium and patients receiving inhaled corticosteroids showed higher frequencies of Fusarium and Mortierella than those in the healthy control group. ${ }^{50}$

Furthermore, hypersensitivity to certain fungal species, such as Aspergillus species, may result in allergic bronchopulmonary aspergillosis (ABPA) and has been reported in poorly controlled asthma. ${ }^{51,52}$

When the BAL mycobiome was examined, Rhodosporidium, Pneumocystis, Leucosporidium, and 
Rhodotorula were more abundant in the asthmatic cohort $(\mathrm{n}=15)$ than in healthy children $(\mathrm{n}=11)$, and Davidiella, Cryptococcus, and Sterigmatomyces were more abundant in healthy children. ${ }^{53}$

Moreover, endotracheal brushes and BAL were collected from 39 patients with asthma to assess the mycobiome composition relative to that of 19 healthy subjects. Fungal diversity was lower in the asthmatic group, with predominant Trichoderma in EB samples and Cladosporium, Fusarium, Aspergillus, and Alternaria in BAL samples. ${ }^{54}$

\section{Intestinal (Gut) Mycobiome}

Compared with bacteria, relatively little is known about the role of fungi within the intestinal microbiota. A study of the enteric microbial burden in 124 healthy adults using Illumina-based metagenomic sequencing demonstrated that over $99 \%$ of the sequence reads originated from bacteria, and the fungal mycobiome occupied less than $0.1 \%$ of the organisms populating the large intestinal tract. $^{55}$

Another study has shown that prolonged antifungal therapy in mice reduces Candida species and increases Aspergillus, Wallemia, and Epicoccum, promoted the development of severe colitis, and exacerbated airway allergies, supporting the major role of fungal species in the stabilization of the gut and airway ecosystems. ${ }^{56}$

A major limitation of these previous studies is the reliance on reference sequences available in GenBank. Fungal sequences are limited and are underrepresented compared with published bacterial genomes, suggesting that gutcolonizing fungal elements may be underdiagnosed.

Although certain fungal genera are commonly found as part of the healthy intestinal ecosystem, such as Candida, increased colonization by Candida itself has been linked to certain inflammatory conditions, such as inflammatory bowel disease. $^{57}$

\section{Virome}

Viral communities represent an essential part of the respiratory ecosystem. However, studies of the resident viral communities in the airways are relatively scarce. Viruses within a microbiome are normally referred to as the virome, virobiome, or viral microbiome and they classically require different approaches for analysis. A shotgun metagenomics approach has been used to analyze viruslike particles. $^{58}$ It is well-documented that the majority of asthma exacerbations are linked to viral infections. ${ }^{38,59,60}$
Although the roles of viruses in the course of respiratory infections are well documented, particularly in the case of influenza viruses and respiratory syncytial virus (RSV), recent studies have addressed a potential link between some viral families, such as Herpesviridae and Anelloviridae, and chronic respiratory conditions, such as asthma. Studies of pediatric asthma have shown that infection with RSV is a risk factor for respiratory morbidities, including asthma, and could worsen the symptoms of asthma. $^{61}$ Additionally, rhinoviruses (RVs) have been linked to asthma exacerbation by acting on mucin hypersecretion in asthmatic airways. ${ }^{62}$ In addition to viruses identified as major risk factors for asthma, other less frequent viruses have been found in children. For example, Human bocavirus (HBoV) was found in the nasopharyngeal aspirates of children $<5$ years old with recurrent wheezing $^{63}$ and the Human metapneumovirus HmpV was identified as a risk factor for asthma. ${ }^{64}$

In an analysis of 134 nasopharyngeal swabs from hospitalized children with acute respiratory infection by whole-genome shotgun sequencing, Rhinovirus $\mathrm{C}$, Bocavirus 1 (BoV), RSV-B, and Parvovirus were relatively more frequent in the asthmatic group than in healthy controls, and the frequencies of Rhinovirus A, Torque teno mini virus (TTMV), and RSV-B were elevated in pneumonia cases. ${ }^{65}$ Torque teno virus (TTV) and RSV-B were found in both groups.

Based on nasopharyngeal swabs of 16 children with asthma, 9 major respiratory virus clusters were detected: human adenovirus (3/16 samples), human RSV (5/16), human rhinovirus $(5 / 16)$, influenza virus $(2 / 16)$, parainfluenza virus (1/16), human BoV (1/16), and human enterovirus (1/16). ${ }^{66}$ Similarly, when 211 nasopharyngeal samples collected from children with asthma exacerbation were tested, viruses were isolated in $20 \%$ of the cohort, particularly rhinovirus, RSV, and enterovirus. ${ }^{67}$

When the respiratory virome was examined in 214 children with asthma at two time points, 33\% of samples collected at the stage of "no respiratory symptoms" contained viruses, of which $64 \%$ were identified as rhinoviruses. $^{68}$ Interestingly, the presence of viruses in baseline samples was not predictive of future asthma exacerbation.

Viral dysbiosis in young children with asthma was also evident in nasopharyngeal aspirates evaluated by a metagenomics approach, manifested by a bacteriophage deficiency and increased eukaryotic viral communities driven by anelloviruses and picornaviruses. ${ }^{69}$ Since 
bacteriophages actively regulate bacterial communities, changes in phage composition may reflect an altered bacterial composition. Such a link has been reported in studies of intestinal inflammatory conditions, such as inflammatory bowel disease; however, to our knowledge, this link has not been tested in allergic respiratory conditions, such as asthma. ${ }^{70}$

\section{Parasitome}

Studies of the metagenomics of parasites or what is referred to as the ""parasitome" or parasite microbiome, are scarce, with limited data available for the gut microbiome only. ${ }^{71}$ Indeed, more studies of the parasitome of the extra-intestinal compartments are needed, particularly studies of the respiratory systems and the progression of asthma. Interestingly, intestinal infection with hookworm is linked to reduced susceptibility to allergic conditions, like asthma. ${ }^{72,73}$ This may be attributed to a symbiotic relationship between helminths and the resident gut microbiota, which improves immune homeostasis in the gut ecosystem. It may also be explained by specific immunomodulatory features of the hookworm, which may have influenced other parts of the body. ${ }^{74}$ Relationships between parasites and microbial taxa have been studied experimentally in a limited number of intestinal conditions, such celiac disease. ${ }^{74}$ The generalizability of the role of the parasitic composition in the airways is yet to be explored. A call for a parasite microbiome project has been issued lately to shed more light on the parasitic composition of the human microbiome and its role in biological processes and diseases. ${ }^{75}$

An assessment of the presence of protozoa in the sputum of adults with asthma and healthy adults $(\mathrm{n}=96)$ revealed a positive relationship between flagellated protozoa and asthma. ${ }^{76}$

\section{Challenges in Microbiome Research}

Despite promising work in microbiome research and substantial advances in our understanding of the association between microbial dysbiosis and the development of allergic conditions, such as asthma, additional studies are needed. In particular, it is necessary to explore the airway microbiome in relation to the pathophysiology, control, and exacerbation of asthma and to determine whether probiotics have beneficial effects.

Microbiome properties may vary among individuals with different risk factors, such as smoking, with the potential to alter the microbial flora. ${ }^{77}$ There is evidence that the oral microbiome, but not the lung microbiome, differs significantly between smokers and non-smokers. ${ }^{77}$ Additionally, the most common genera in both the oral and lung microbiomes were Streptococcus, Prevotella, and Veillonella; however, Haemophilus and Enterobacteriaceae were more abundant in the lung microbiome. ${ }^{77}$

One major limitation of studies of the asthma-microbiome relationship is that the general focus on a single time point in cross-sectional studies. This is contrary to the nature of the human microbiome, which is dynamic and changes in response to many factors contributing to its diversity, including environmental and biological factors. A randomized, longitudinal analysis of the nasal microbiome involving 214 children with asthma found a significant difference in the airway microbiome between younger (5-7 years old) and older children (8-11 years). ${ }^{68}$ A switch from a Corynebacterium + Dolosigranulum fingerprint towards abundant Moraxella was also noted between the two time points in children with "no respiratory symptoms" to ""early signs of exacerbated asthma," reflecting the instability of the microbiome and its close association with disease manifestation. Another major limitation is that the link between the microbial signature and the pathophysiological mechanism underlying asthma may be confounded by the asthma endotype and the inflammatory process.

Another limitation of studies of the asthma microbiome is the focus on a single target or site. Since the majority of chronic inflammatory disorders are multifactorial in nature with multiple organs involvement, the microbiome fingerprint across the affected systems may be altered, in addition to the intestinal microbiome, which is frequently used as a biomarker for respiratory allergies. Therefore, analyses of multiple sample types or organs, including the gut, would yield more clinically meaningful data. A previous study has assessed microbial diversity and quantity at multiple sites, including the mouth, nose, lung, and stomach in healthy individuals. ${ }^{11}$ Interestingly, sequencing of 16S rRNA amplicon libraries demonstrated that the oral cavity and gastric fluid had the highest microbial burdens with comparable diversity (similar), suggesting a constant trafficking channel along the oral-gastric axis. The lowest microbial burden was noted in the nasal cavities and lung, although microbial similarity was reported between the oral and lung microbiota and not the nose flora. It should be noted that the gastric fluid may have been contaminated by the oral flora and that inter-subject variation is likely, as risk factors vary significantly among healthy individuals. ${ }^{11}$ 
Although studies of the asthmatic microbiome have adopted certain inclusion and exclusion criteria, other confounding factors may interfere with the results, such as therapeutic interventions (including corticosteroids and/or antibiotics) and the presence of undisclosed food intolerances, as all of these factors either directly contribute to the composition of the respiratory microbiome or indirectly influence the composition via alterations in the intestinal microbiota. ${ }^{78}$

Sampling error and complexity of microbiome analysis techniques also represent a challenge.$^{79}$ Majority of microbiota profiling technology (Figure 1) are complex and require sophisticated computational programs. In addition, analysis of metagenomics data focuses mostly on genes with known biological functions which limits the discovery of novel, potentially significant sequences. ${ }^{79}$

\section{Conclusions and Future Perspectives}

Asthma is the most prevalent pediatric chronic disease and affects more than 300 million people worldwide. In the last few years, microbiome studies, particularly studies of the gut microbiome, have pioneered research aimed at identifying the root cause of asthmatic airways and hyperresponsiveness. These studies have revealed a distinct microbial community that naturally populates the gut and the respiratory system and plays a major role in modulating the homeostasis of the airways. Disturbances in the microbial ecosystem are directly linked to the development of asthma and further to asthma exacerbation (Figure 2).

The scope of microbiome studies, irrespective of approach (pyrosequencing or shotgun metagenomics) has largely been limited to the relative abundance of bacterial taxa, with far less focus on other microorganisms. To move beyond microbial level analyses and gain a more comprehensive overview of the determinants of asthma, other advanced techniques, best known as ""omics" techniques are needed. More studies are needed to assess the microbiota at the transcriptome level and to determine which genes are actively expressed within the airways. Proteomics and metabolomics could provide a better understanding of the functional aspects of microbial dysbiosis based on metabolites released by the resident microbiota and their associations with hypersensitivity. For example, metabolomics analyses of the intestinal microbiota in asthma have shown that reductions in certain bacterial taxa are coupled with decreased levels of fecal acetate and the disruption of enterohepatic metabolites. ${ }^{17}$ To the best of our knowledge, such an experimental approach has not been applied to pulmonary samples in patients with asthma.

Despite the focus on bacterial communities, the contributions of other microorganisms, such as fungi, viruses, and archaea, cannot be ruled out. Therefore, more studies are needed to understand the non-bacterial microbiome inhabitants. This review provides an updated perspective of the airway microbiome during childhood and summarize our understanding of microbial changes during development, providing a basis for the establishment of predictive biomarkers for a predisposition to allergies. In the era of COVID-19, analyses of respiratory microbial communities are of a great importance and can provide a valuable tool for improving our understanding of local interactions between airway compartments, populating microorganisms, and the immune status. These studies may lead to the development of microbe-based diagnostics and therapies, potentially in the form of probiotics, to prevent the development of asthma and related allergic diseases.

\section{Abbreviations}

BAL, bronchoalveolar lavage; CRS, chronic rhinosinusitis; COPD, chronic obstructive pulmonary disease; BPD, bronchopulmonary dysplasia; ABPA, Allergic bronchopulmonary aspergillosis; EB, endotracheal brushes; ITS, internal transcribed spacer.

\section{Acknowledgment}

The author extends her appreciation to the Deputyship for Research\& Innovation, Ministry of Education in Saudi Arabia for funding this research work through the project number (IF-2020-016-CAMS) at Imam Abdulrahman bin Faisal University/College of Applied Medical Sciences.

\section{Disclosure}

The author reports no conflicts of interest in this work.

\section{References}

1. Dharmage SC, Perret JL, Custovic A. Epidemiology of asthma in children and adults. Front Pediatr. 2019;7:246. doi:10.3389/fped. 2019.00246

2. Toskala E, Kennedy DW. Asthma risk factors. Int Forum Allergy Rhinol. 2015;5(Suppl 1):S11-6. doi:10.1002/alr.21557 
3. Abdel-Aziz MI, Vijverberg SJH, Neerincx AH, Kraneveld AD, Maitland-van der Zee AH. The crosstalk between microbiome and asthma: exploring associations and challenges. Clin Exp Allergy. 2019;49(8):1067-1086. doi:10.1111/cea.13444

4. Ursell LK, Metcalf JL, Parfrey LW, Knight R. Defining the human microbiome. Nutr Rev. 2012;70(Suppl 1):S38-44. doi:10.1111/ j.1753-4887.2012.00493.x

5. Kelly RS, Dahlin A, McGeachie MJ, et al. Asthma metabolomics and the potential for integrative omics in research and the clinic. Chest. 2017;151(2):262-277. doi:10.1016/j.chest.2016.10.008

6. Hilton SK, Castro-Nallar E, Pérez-Losada M, et al. Metataxonomic and metagenomic approaches vs. culture-based techniques for clinical pathology. Front Microbiol. 2016;7:484. doi:10.3389/fmicb.2016.00484

7. Quince C, Walker AW, Simpson JT, Loman NJ, Segata N. Shotgun metagenomics, from sampling to analysis. Nat Biotechnol. 2017;35 (9):833-844. doi:10.1038/nbt.3935

8. Chakravorty S, Helb D, Burday M, Connell N, Alland D. A detailed analysis of $16 \mathrm{~S}$ ribosomal RNA gene segments for the diagnosis of pathogenic bacteria. J Microbiol Methods. 2007;69(2):330-339. doi:10.1016/j.mimet.2007.02.005

9. Ranjan R, Rani A, Metwally A, McGee HS, Perkins DL. Analysis of the microbiome: advantages of whole genome shotgun versus $16 \mathrm{~S}$ amplicon sequencing. Biochem Biophys Res Commun. 2016;469 (4):967-977. doi:10.1016/j.bbrc.2015.12.083

10. Castillo DJ, Rifkin RF, Cowan DA, Potgieter M. The healthy human blood microbiome: fact or fiction? Front Cell Infect Microbiol. 2019;9:148. doi:10.3389/fcimb.2019.00148

11. Bassis CM, Erb-Downward JR, Dickson RP, et al. Analysis of the upper respiratory tract microbiotas as the source of the lung and gastric microbiotas in healthy individuals. mBio. 2015;6(2):e00037. doi: $10.1128 / \mathrm{mBio} .00037-15$

12. Okba AM, Saber SM, Abdel-Rehim AS, Amin MM, Mohamed DA. Fecal microbiota profile in atopic asthmatic adult patients. Eur Ann Allergy Clin Immunol. 2018;50(3):117-124. doi:10.23822/ EurAnnACI.1764-1489.48

13. Moffatt MF, Cookson WO. The lung microbiome in health and disease. Clin Med (Lond). 2017;17(6):525-529. doi:10.7861/clinmedicine. 17-6-525

14. Faner R, Sibila O, Agustí A, et al. The microbiome in respiratory medicine: current challenges and future perspectives. Eur Respir J. 2017;49. doi:10.1183/13993003.02086-2016

15. Jones HE, Harris KA, Azizia M, et al. Differing prevalence and diversity of bacterial species in fetal membranes from very preterm and term labor. PLoS One. 2009;4(12):e8205. doi:10.1371/journal. pone. 0008205

16. Hansen R, Scott KP, Khan S, et al. First-pass meconium samples from healthy term vaginally-delivered neonates: an analysis of the microbiota. PLoS One. 2015;10(7):e0133320. doi:10.1371/journal. pone. 0133320

17. Arrieta MC, Stiemsma LT, Dimitriu PA, et al. Early infancy microbial and metabolic alterations affect risk of childhood asthma. Sci Transl Med. 2015;7(307):307ra152. doi:10.1126/scitranslmed.aab2271

18. Lal CV, Travers C, Aghai ZH, et al. The airway microbiome at birth. Sci Rep. 2016;6:31023. doi:10.1038/srep31023

19. Aagaard K, Ma J, Antony KM, Ganu R, Petrosino J, Versalovic J. The placenta harbors a unique microbiome. Sci Transl Med. 2014;6 (237):237ra65. doi:10.1126/scitranslmed.3008599

20. Mourani PM, Harris JK, Sontag MK, Robertson CE, Abman SH. Molecular identification of bacteria in tracheal aspirate fluid from mechanically ventilated preterm infants. PLoS One. 2011;6(10): e25959. doi:10.1371/journal.pone.0025959

21. Lohmann P, Luna RA, Hollister EB, et al. The airway microbiome of intubated premature infants: characteristics and changes that predict the development of bronchopulmonary dysplasia. Pediatr Res. 2014;76(3):294-301. doi:10.1038/pr.2014.85
22. Mika M, Mack I, Korten I, et al. Dynamics of the nasal microbiota in infancy: a prospective cohort study. $J$ Allergy Clin Immunol. 2015;135(4):905-12.e11. doi:10.1016/j.jaci.2014.12.1909

23. Biesbroek G, Tsivtsivadze E, Sanders EA, et al. Early respiratory microbiota composition determines bacterial succession patterns and respiratory health in children. Am J Respir Crit Care Med. 2014;190 (11):1283-1292. doi:10.1164/rccm.201407-1240OC

24. van Tilburg Bernardes E, Gutierrez MW, Arrieta MC. The fungal microbiome and asthma. Front Cell Infect Microbiol. 2020;10:583418. doi:10.3389/fcimb.2020.583418

25. Dzidic M, Abrahamsson TR, Artacho A, Collado MC, Mira A, Jenmalm MC. Oral microbiota maturation during the first 7 years of life in relation to allergy development. Allergy. 2018;73(10):20 00-2011. doi:10.1111/all.13449

26. Depner M, Ege MJ, Cox MJ, et al. Bacterial microbiota of the upper respiratory tract and childhood asthma. J Allergy Clin Immunol. 2017;139(3):826-834.e13. doi:10.1016/j.jaci.2016.05.050

27. Espuela-Ortiz A, Lorenzo-Diaz F, Baez-Ortega A, et al. Bacterial salivary microbiome associates with asthma among african american children and young adults. Pediatr Pulmonol. 2019;54(12): 1948-1956. doi:10.1002/ppul.24504

28. Boutin S, Depner M, Stahl M, et al. Comparison of oropharyngeal microbiota from children with asthma and cystic fibrosis. Mediators Inflamm. 2017;2017:5047403. doi:10.1155/2017/5047403

29. Ramakrishnan VR, Hauser LJ, Feazel LM, Ir D, Robertson CE, Frank DN. Sinus microbiota varies among chronic rhinosinusitis phenotypes and predicts surgical outcome. J Allergy Clin Immunol. 2015;136(2):334-42.e1. doi:10.1016/j.jaci.2015.02.008

30. Toivonen L, Karppinen S, Schuez-Havupalo L, et al. Longitudinal changes in early nasal microbiota and the risk of childhood asthma. Pediatrics. 2020;146. doi:10.1542/peds.2020-0421

31. Mahdavinia M, Engen PA, LoSavio PS, et al. The nasal microbiome in patients with chronic rhinosinusitis: analyzing the effects of atopy and bacterial functional pathways in 111 patients. J Allergy Clin Immunol. 2018;142(1):287-290.e4. doi:10.1016/j. jaci.2018.01.033

32. Atarashi K, Tanoue T, Oshima K, et al. Treg induction by a rationally selected mixture of Clostridia strains from the human microbiota. Nature. 2013;500(7461):232-236. doi:10.1038/nature12331

33. Pérez-Losada M, Authelet KJ, Hoptay CE, Kwak C, Crandall KA, Freishtat RJ. Pediatric asthma comprises different phenotypic clusters with unique nasal microbiotas. Microbiome. 2018;6(1):179. doi:10. 1186/s40168-018-0564-7

34. Lee JJ, Kim SH, Lee MJ, et al. Different upper airway microbiome and their functional genes associated with asthma in young adults and elderly individuals. Allergy. 2019;74(4):709-719. doi:10.1111/ all.13608

35. Yang HJ, LoSavio PS, Engen PA, et al. Association of nasal microbiome and asthma control in patients with chronic rhinosinusitis. Clin Exp Allergy. 2018;48(12):1744-1747. doi:10.1111/cea.13255

36. Fazlollahi M, Lee TD, Andrade J, et al. The nasal microbiome in asthma. J Allergy Clin Immunol. 2018;142(3):834-843.e2. doi:10.10 16/j.jaci.2018.02.020

37. Gleeson K, Eggli DF, Maxwell SL. Quantitative aspiration during sleep in normal subjects. Chest. 1997;111(5):1266-1272. doi:10.13 78/chest.111.5.1266

38. O'Dwyer DN, Dickson RP, Moore BB. The lung microbiome, immunity, and the pathogenesis of chronic lung disease. $J$ Immunol. 2016;196(12):4839-4847. doi:10.4049/jimmunol.1600279

39. Dickson RP, Erb-Downward JR, Freeman CM, et al. Spatial variation in the healthy human lung microbiome and the adapted island model of lung biogeography. Ann Am Thorac Soc. 2015;12(6):821-830. doi:10.1513/AnnalsATS.201501-029OC

40. Hilty M, Burke C, Pedro H, et al. Disordered microbial communities in asthmatic airways. PLoS One. 2010;5(1):e8578. doi:10.1371/journal.pone. 0008578 
41. Marri PR, Stern DA, Wright AL, Billheimer D, Martinez FD Asthma-associated differences in microbial composition of induced sputum. J Allergy Clin Immunol. 2013;131(2):346-52.e1-3. doi:10.10 16/j.jaci.2012.11.013

42. Budden KF, Gellatly SL, Wood DL, et al. Emerging pathogenic links between microbiota and the gut-lung axis. Nat Rev Microbiol. 2017;15(1):55-63. doi:10.1038/nrmicro.2016.142

43. Denning DW, O'Driscoll BR, Hogaboam CM, Bowyer P, Niven RM. The link between fungi and severe asthma: a summary of the evidence. Eur Respir J. 2006;27(3):615-626. doi:10.1183/090319 36.06.00074705

44. Ghannoum MA, Jurevic RJ, Mukherjee PK, et al. Characterization of the oral fungal microbiome (mycobiome) in healthy individuals. PLoS Pathog. 2010;6(1):e1000713. doi:10.1371/journal.ppat.1000713

45. Jung WH, Croll D, Cho JH, Kim YR, Lee YW. Analysis of the nasal vestibule mycobiome in patients with allergic rhinitis. Mycoses. 2015;58(3):167-172. doi:10.1111/myc. 12296

46. Sellart-Altisent M, Torres-Rodríguez JM, Gómez de Ana S, Alvarado-Ramírez E. [Nasal fungal microbiota in allergic and healthy subjects]. Rev Iberoam Micol. 2007;24(2):125-130. Spanish. doi:10.1016/s1130-1406(07)70027-x

47. Weaver D, Gago S, Bromley M, Bowyer P. The human lung mycobiome in chronic respiratory disease: limitations of methods and our current understanding. Curr Fungal Infect Rep. 2019;13(3):109-119. doi:10.1007/s12281-019-00347-5

48. van Woerden HC, Gregory C, Brown R, Marchesi JR, Hoogendoorn B, Matthews IP. Differences in fungi present in induced sputum samples from asthma patients and non-atopic controls: a community based case control study. BMC Infect Dis. 2013;13:69. doi:10.1186/1471-2334-13-69

49. Fraczek MG, Chishimba L, Niven RM, et al. Corticosteroid treatment is associated with increased filamentous fungal burden in allergic fungal disease. J Allergy Clin Immunol. 2018;142(2):407-414. doi:10.1016/j.jaci.2017.09.039

50. Huang $\mathrm{C}, \mathrm{Yu} \mathrm{Y,} \mathrm{Du} \mathrm{W,} \mathrm{et} \mathrm{al.} \mathrm{Fungal} \mathrm{and} \mathrm{bacterial} \mathrm{microbiome}$ dysbiosis and imbalance of trans-kingdom network in asthma. Clin Transl Allergy. 2020;10:42. doi:10.1186/s13601-020-00345-8

51. Agarwal R, Chakrabarti A, Shah A, et al. Allergic bronchopulmonary aspergillosis: review of literature and proposal of new diagnostic and classification criteria. Clin Exp Allergy. 2013;43(8):850-873. doi:10.1111/cea.12141

52. Underhill DM, Iliev ID. The mycobiota: interactions between commensal fungi and the host immune system. Nat Rev Immunol. 2014;14(6):405-416. doi:10.1038/nri3684

53. Goldman DL, Chen Z, Shankar V, Tyberg M, Vicencio A, Burk R. Lower airway microbiota and mycobiota in children with severe asthma. J Allergy Clin Immunol. 2018;141(2):808-811.e7. doi:10. 1016/j.jaci.2017.09.018

54. Sharma A, Laxman B, Naureckas ET, et al. Associations between fungal and bacterial microbiota of airways and asthma endotypes. J Allergy Clin Immunol. 2019;144(5):1214-1227.e7. doi:10.1016/j. jaci.2019.06.025

55. Qin J, Li R, Raes J, et al. A human gut microbial gene catalogue established by metagenomic sequencing. Nature. 2010;464(7285): 59-65. doi:10.1038/nature08821

56. Wheeler ML, Limon JJ, Bar AS, et al. Immunological Consequences of Intestinal Fungal Dysbiosis. Cell Host Microbe. 2016;19 (6):865-873. doi:10.1016/j.chom.2016.05.003

57. Standaert-Vitse A, Jouault T, Vandewalle P, et al. Candida albicans is an immunogen for anti-Saccharomyces cerevisiae antibody markers of Crohn's disease. Gastroenterology. 2006;130(6):1764-1775. doi:10.1053/j.gastro.2006.02.009

58. Hurwitz BL, Deng L, Poulos BT, Sullivan MB. Evaluation of methods to concentrate and purify ocean virus communities through comparative, replicated metagenomics. Environ Microbiol. 2013;15 (5):1428-1440. doi:10.1111/j.1462-2920.2012.02836.x
59. Johnston SL, Pattemore PK, Sanderson G, et al. Community study of role of viral infections in exacerbations of asthma in 9-11 year old children. BMJ. 1995;310(6989):1225-1229. doi:10.1136/bmj.310.69 89.1225

60. Busse WW, Lemanske RF, Gern JE. Role of viral respiratory infections in asthma and asthma exacerbations. Lancet. 2010;376 (9743):826-834. doi:10.1016/S0140-6736(10)61380-3

61. Jankauskaitė L, Misevičienė V, Vaidelienė L, Kèvalas R. Lower airway virology in health and disease-from invaders to symbionts. Medicina (Kaunas). 2018;54. doi:10.3390/medicina54050072.

62. Hewson CA, Haas JJ, Bartlett NW, et al. Rhinovirus induces MUC5AC in a human infection model and in vitro via NF- $\mathrm{KB}$ and EGFR pathways. Eur Respir J. 2010;36(6):1425-1435. doi:10.1183/ 09031936.00026910

63. Deerojanawong J, Satdhabudha A, Prapphal N, Sritippayawan S, Samransamruajkit R. Incidence of recurrent wheezing in under 5 -year-old human bocavirus infection during one year follow-up. J Med Assoc Thai. 2013;96(2):185-191.

64. García-García ML, Calvo C, Casas I, et al. Human metapneumovirus bronchiolitis in infancy is an important risk factor for asthma at age 5. Pediatr Pulmonol. 2007;42(5):458-464. doi:10.1002/ppul.20597

65. Romero-Espinoza JA, Moreno-Valencia Y, Coronel-Tellez RH, et al. Virome and bacteriome characterization of children with pneumonia and asthma in Mexico City during winter seasons 2014 and 2015. PLoS One. 2018;13(2):e0192878. doi:10.1371/journal.pone.0192878

66. Yang J, Yang F, Ren L, et al. Unbiased parallel detection of viral pathogens in clinical samples by use of a metagenomic approach. J Clin Microbiol. 2011;49(10):3463-3469. doi:10.1128/JCM.00273-11

67. Robledo Aceves M, Barrón Balderas A, Jaime Ornelas ML. Most commonly isolated viruses in asthma exacerbation and their correlation with eosinophil and total serum immunoglobulin E levels. Arch Argent Pediatr. 2018;116(3):192-197. doi:10.5546/aap.2018.eng.192

68. Zhou Y, Jackson D, Bacharier LB, et al. The upper-airway microbiota and loss of asthma control among asthmatic children. Nat Commun. 2019;10(1):5714. doi:10.1038/s41467-019-13698-x

69. Spyridon Megremis BC, Xepapadaki P, Bachert C, et al. Bacteriophage deficiency characterizes respiratory virome dysbiosis in childhood asthma. bioRxiv. 2020;08:04.236067.

70. Sinha A, Maurice CF. Bacteriophages: uncharacterized and dynamic regulators of the immune system. Mediators Inflamm. 2019;20 19:3730519. doi:10.1155/2019/3730519

71. Marzano V, Mancinelli L, Bracaglia G, et al. "Omic" investigations of protozoa and worms for a deeper understanding of the human gut "parasitome. PLoS Negl Trop Dis. 2017;11(11):e0005916. doi:10. 1371/journal.pntd.0005916

72. Loukas A, Hotez PJ, Diemert D, et al. Hookworm infection. Nat Rev Dis Primers. 2016;2:16088. doi:10.1038/nrdp.2016.88

73. Cooper PJ. Interactions between helminth parasites and allergy. Curr Opin Allergy Clin Immunol. 2009;9(1):29-37. doi:10.1097/ACI.0b0 13e32831f44a6

74. Giacomin P, Zakrzewski M, Croese J, et al. Experimental hookworm infection and escalating gluten challenges are associated with increased microbial richness in celiac subjects. Sci Rep. 2015;5: 13797. doi: $10.1038 /$ srep 13797

75. Dheilly NM, Bolnick D, Bordenstein S, et al. Parasite microbiome project: systematic investigation of microbiome dynamics within and across parasite-host interactions. mSystems. 2017;2(4). doi:10.1128/ mSystems.00050-17

76. van Woerden HC, Ratier-Cruz A, Aleshinloye OB, Martinez-Giron R, Gregory C, Matthews IP. Association between protozoa in sputum and asthma: a case-control study. Respir Med. 2011;105(6):877-884. doi:10.1016/j.rmed.2010.11.016

77. Morris A, Beck JM, Schloss PD, et al. Comparison of the respiratory microbiome in healthy nonsmokers and smokers. Am J Respir Crit Care Med. 2013;187(10):1067-1075. doi:10.1164/rccm.201210$19130 \mathrm{OC}$ 
78. Love BL, Mann JR, Hardin JW, Lu ZK, Cox C, Amrol DJ. Antibiotic prescription and food allergy in young children. Allergy Asthma Clin Immunol. 2016;12:41. doi:10.1186/s13223-016-0148-7
79. Sergaki C, Lagunas B, Lidbury I, Gifford ML, Schäfer P. Challenges and approaches in microbiome research: from fundamental to applied. Front Plant Sci. 2018;9:1205. doi:10.3389/fpls.2018.01205

\section{Publish your work in this journal}

The International Journal of General Medicine is an international, peer-reviewed open-access journal that focuses on general and internal medicine, pathogenesis, epidemiology, diagnosis, monitoring and treatment protocols. The journal is characterized by the rapid reporting of reviews, original research and clinical studies across all disease areas. The manuscript management system is completely online and includes a very quick and fair peer-review system, which is all easy to use. Visit http://www.dovepress.com/ testimonials.php to read real quotes from published authors. 